\title{
Vacquinol-1 induces apoptosis in hepatocellular carcinoma cell
}

\author{
SHU-LAN SUN ${ }^{1 *}$, XIAOXI LI $^{1 *}$, NAN SU $^{1}$, SHI CHEN $^{1}$, XIAOXIN GAO $^{1}$, \\ GUIRONG ZHANG ${ }^{1}$ and HAOZHE PIAO ${ }^{1,2}$ \\ ${ }^{1}$ Central Laboratory; ${ }^{2}$ Department of Neurosurgery, Cancer Hospital of China Medical University, \\ Liaoning Cancer Hospital and Institute, Shenyang, Liaoning 110042, P.R. China
}

Received September 25, 2017; Accepted April 20, 2018

DOI: $10.3892 / \mathrm{mmr} .2018 .8957$

\begin{abstract}
Vacquinol-1 (Vacq), a quinolone derivative, has recently been reported to display potent antitumor effects in glioblastomas by inducing cellular massive vacuolization and cell death. However, whether Vacq induces cytotoxicities in other types of cancers, and the potential underlying mechanism, remain to be investigated. In the present study, it was revealed that Vacq suppressed cell growth and colony formation in the hepatocellular carcinoma (HCC) cell lines BEL7402 and Huh7. In addition, treatment with Vacq increased the number of early and late apoptotic cells as assessed by flow cytometry with fluorescein isothiocyanate-conjugated Annexin $\mathrm{V}$ and propidium iodide double staining. Notably, the effect by Vacq in the tested cells could be inhibited by pretreatment with a broad specificity caspase inhibitor Z-VAD-FMK, suggesting that Vacq may induce apoptosis in HCC cells. Morphologically, exposure to Vacq resulted in nuclear fragmentation and the apoptotic body formation in HCC cells. Furthermore, Vacq treatment increased the cleavage of caspase-3, caspase-9 and poly(adenosine diphosphate-ribose) polymerase-1. Mechanistic analysis revealed that Vacq upregulated the expressions of pro-apoptotic proteins [B-cell lymphoma 2 (bcl-2)-associated X protein (Bax) and Bcl-2-like protein 11] and downregulated the pro-survival
\end{abstract}

Correspondence to: Professor Haozhe Piao, Central Laboratory, Cancer Hospital of China Medical University, Liaoning Cancer Hospital and Institute, 44 Xiaoheyan Road, Dadong, Shenyang, Liaoning 110042, P.R. China

E-mail: sunshulan@cancerhosp-ln-cmu.com

${ }^{*}$ Contributed equally

Abbreviations: Vacq, Vacquinol-1; HCC, Hepatocellular carcinoma; GS, glioblastomas cell; DMSO, dimethyl sulfoxide; FITC, fluorescein isothiocyanate; PI, propidium iodide; CD-DST, collagen gel droplet-embedded culture drug sensitivity test; Dox, Doxorubicin; PARP-1, poly(adenosine diphosphate-ribose) polymerase-1

Key words: apoptosis, B-cell lymphoma 2 family proteins, B-cell lymphoma 2-associated $\mathrm{X}$ protein translocation, collagen gel droplet-embedded culture drug sensitivity test, hepatocellular carcinoma, Vacquinol-1 protein, Bcl-2, expression in HCC cells. Furthermore, Vacq induced Bax translocation. Of note, Vacq displayed inhibitory effects on patient-derived HCC cells in two-dimensional (2D) and three-dimensional (3D) cultures. Taken together, the data suggested that Vacq induced intrinsic apoptosis and may be utilized as an effective reagent for HCC treatment.

\section{Introduction}

Hepatocellular carcinoma (HCC) is the most common type of primary liver cancer. The incidence of HCC keeps increasing in recent years (1). Surgery, chemotherapies and radiotherapy are the conventional treatments for HCC. However, late diagnosis along with resistance to chemotherapies relate to the limited clinical efficacy (2). Therefore, there is an urgent need to develop effective agents for $\mathrm{HCC}$ treatment.

Vacquinol-1 (Vacq) is a quinolone derivative which shows antitumor effects in glioblastomas (3). Vacq rapidly induced glioblastomas cells (GCs) death and catastrophic vacuolization by membrane ruffling, cell rounding, massive macropinocytic vacuole accumulation, ATP depletion, and cytoplasmic membrane rupture of GCs, which was morphologically distinct from apoptosis (3). Currently, whether Vacq could display antitumor effects in other types of cancer remains to be examined. In addition, whether Vacq could induce other types of cell death besides catastrophic vacuolization in cancer cells needs to be further investigated.

In the present study, we aimed to investigate the effects of Vacq on human HCC cells. Distinct from observations in previous study, we showed that Vacq triggered caspase dependent apoptosis in HCC cells.

\section{Materials and methods}

Cell lines. HCC cell lines BEL7402 and Huh7 were provided by Cell Bank of the Chinese Academy of Sciences (Shanghai, China). Huh7 was cultured in DMEM high glucose, and BEL7402 was maintained in RPMI1640 (Hyclone; GE Healthcare, Logan UT, UTA). All the cell culture medium was supplemented with 10\% fetal bovine serum (Gibco; Thermo Fisher Scientific, Inc., Waltham, MA, USA). Cells were cultured in a humidified incubator containing $5 \% \mathrm{CO}_{2}$ at $37^{\circ} \mathrm{C}$.

Patients. This study was approved by the Medical Ethical Committee of the Liaoning Cancer Hospital (China; Ethics 
Review approval no. 20160213-3). Written informed consent was provided by all of the participants. Ten patients who were pathologically diagnosed as primary HCC were enrolled in this study. Patients who received chemotherapy and radiotherapy or interventional therapy were exclude from this study.

Compounds and antibodies. Vacq was purchased from Sigma Aldrich (St. Louis, MO, USA) and dissolved in sterile dimethyl sulfoxide (DMSO). Antibodies against caspase-3, caspase-8, caspase-9, BCL-xL, B-cell lymphoma 2 (bcl-2)-associated $\mathrm{X}$ protein (Bax), Bim, and $\beta$-Actin were purchased from Cell Signaling Technology Inc. (Danvers, MA, USA). BCL-2 antibody was obtained from Thermo Fisher Scientific, Inc. The following antibodies were purchased from Abcam (Cambridge MA, USA): Anti-PARP, Goat anti-mouse IgG-HRP and Goat anti-Rabbit IgG-HRP. Caspase inhibitor Z-VAD-FMK were purchased from Promega (Madison, WI, USA). Hoechst 33342 was from Solarbio Science \& Technology (Beijing, China).

Cell viability assay. Cell viability was monitored by $\mathrm{xCEL}-$ Ligence Real-Time Cell Analyzer (RTCA)-MP system (Acea Biosciences, San Diego, CA, USA). This device was put in $5 \% \mathrm{CO}_{2}$ incubator and could measure cellular growth status in real time. Briefly, $100 \mu \mathrm{l}$ of culture medium was added in each well of E-Plate 96 (Acea Biosciences) to obtain equilibrium. $2 \times 10^{4}$ cells for cell line cells or $1 \times 10^{5}$ for human primary HCC cells in $100 \mu 1$ of culture medium were seeded in E-Plate 96, which was coated by biocompatible microelectrode. After $18 \mathrm{~h}$, cells were treated with $25,12.5,6.26,3.13$ and $1.56 \mu \mathrm{M}$, respectively. DMSO-treated cells were used as controls. Electrical impedance which reflects cell growth status was presented as cell index and was read automatically every $1 \mathrm{~min}$. The half maximal inhibitory concentration (IC50) values at 24, 48 and $72 \mathrm{~h}$ were calculated automatically by RTCA software v. 2.0 (Acea Biosciences).

Colony formation. BEL7402 and Huh7 cells were seeded in 6-well culture plates at the density of 2,000 cells/well. Cells were cultured for $18 \mathrm{~h}$ and then treated with different concentrations of Vacq for $24 \mathrm{~h}$. Cells were then washed twice with PBS and cultured for 14 days. Cells were fixed with formalin for $20 \mathrm{~min}$ and stained with crystal violet for $15 \mathrm{~min}$.

Apoptosis assay. Apoptosis was analyzed by flow cytometry (BD Accuri ${ }^{\mathrm{TM}}$ C6; BD Biosciences Franklin Lakes, NJ, USA). Briefly, cells were harvested and washed twice with cold PBS. Collected cells were stained with Annexin V-fluorescein isothiocyanate (FITC) and propidium iodide (PI) and incubated at room temperature for $10 \mathrm{~min}$.

Nuclear morphological observation. $1 \times 10^{4}$ BEL7402 cells were seeded in 6-well plates and treated with Vacq at $10 \mu \mathrm{M}$ or Dox at $2 \mu \mathrm{M}$ for $24 \mathrm{~h}$, respectively. Cells were stained with Hoechst 33342 for $30 \mathrm{~min}$ at $37^{\circ} \mathrm{C}$. After staining, cells were washed with PBS for 3 times and observed with Leica fluorescent microscope (DMi8; Leica Microsystems, Inc., Buffalo Grove, IL, USA).
Western blot analysis. BEL7402 cells were seeded in 6-well culture plates with the density at $3 \times 10^{5}$ cells/well. Cells were exposed to $10 \mu \mathrm{M}$ Vacq for $24 \mathrm{~h}$ and then processed for western blot analysis as previously described (4). The primary antibodies were used at 1:1,000 dilution and incubated at $4^{\circ} \mathrm{C}$ for overnight, and subsequently incubated with secondary antibodies (Goat anti-mouse IgG-HRP, 1:10,000; and Goat anti-Rabbit IgG-HRP, 1:15,000) for $2 \mathrm{~h}$ at room temperature. Immunodetection was performed using Supersignal West Pico plus (Thermo Fisher Scientific, Inc.) and detected by BIO-RAD GelDoc XR+ (Bio-Rad, Berkeley, CA, USA).

Bax translocation. BEL7402 cells were exposed to $10 \mu \mathrm{M}$ Vacq for different durations (0, 1, 3, 6, 9, $12 \mathrm{~h})$. Cytosolic and mitochondrial proteins were extracted by using Cytoplasmic and Mitochondrial Protein Extraction kit (Sangon Biotech, Shanghai, China) according to the manufacturer's instructions. Mitochondrial and cytosolic Bax expressions were detected by western blot analysis. COX IV and $\beta$-Actin were used as mitochondrial and cytosolic loading control, respectively.

Primary HCC cell isolation. Fresh liver cancer specimen obtained from surgery was finely minced and digested with cell dispersion enzyme solution (EZ; Nitta Gelatin Inc., Osaka, Japan) for $30 \mathrm{~min}$. T-he dissociated cancer cells were collected and filtered through an $80 \mu \mathrm{m}$ nylon mesh. Primary cells were cultured in a collagen gel coated flask (CG-flask; Nitta Gelatin Inc.) in DMEM/F12 (Gibco; Thermo Fisher Scientific, Inc.) supplemented with $10 \%$ fetal bovine serum at $37^{\circ} \mathrm{C} 5 \% \mathrm{CO}_{2}$ for $24 \mathrm{~h}$. Only the viable cells adhering to the collagen gel were collected for the following use.

Primary HCC cell sensitivity test. One part of the primary HCC cells was used for cell viability test monitored by xCELLigence RTCA. The rest part of the cells was performed using collagen gel droplet-embedded CD-DST followed by the standard procedure (5). Briefly, HCC cells were cultured in the collagen droplet which provided a 3-D environment for HCC cells. $15 \mu \mathrm{M}$ Vacq was used for treatment. After $24 \mathrm{~h}$, medium containing Vacq was removed and changed to the prepared culture media 2 (PCM-2; Kurabo, Oosaka, Japan) without FBS for 7 days. Viable cells were stained with neutral red, fixed with $10 \%$ neutral buffered formalin, washed in water, air dried, and quantified by optical density image analysis using the Primage System (Solution Systems, Tokyo, Japan).

Statistical analysis. Statistical analysis was performed using one-way analysis of variance and SPSS version 21 software (IBM Corp., Armonk, NY, USA). Multiple comparisons between treatment groups and controls were evaluated using Dunnett's least significant difference test. $\mathrm{P}<0.05$ was considered to indicate a statistically significant difference. All experiments were carried out in triplicate in three independent experiments.

\section{Results}

Vacq suppresses human HCC cell proliferation and colony formation. To determine the effect of Vacq on HCC cell 

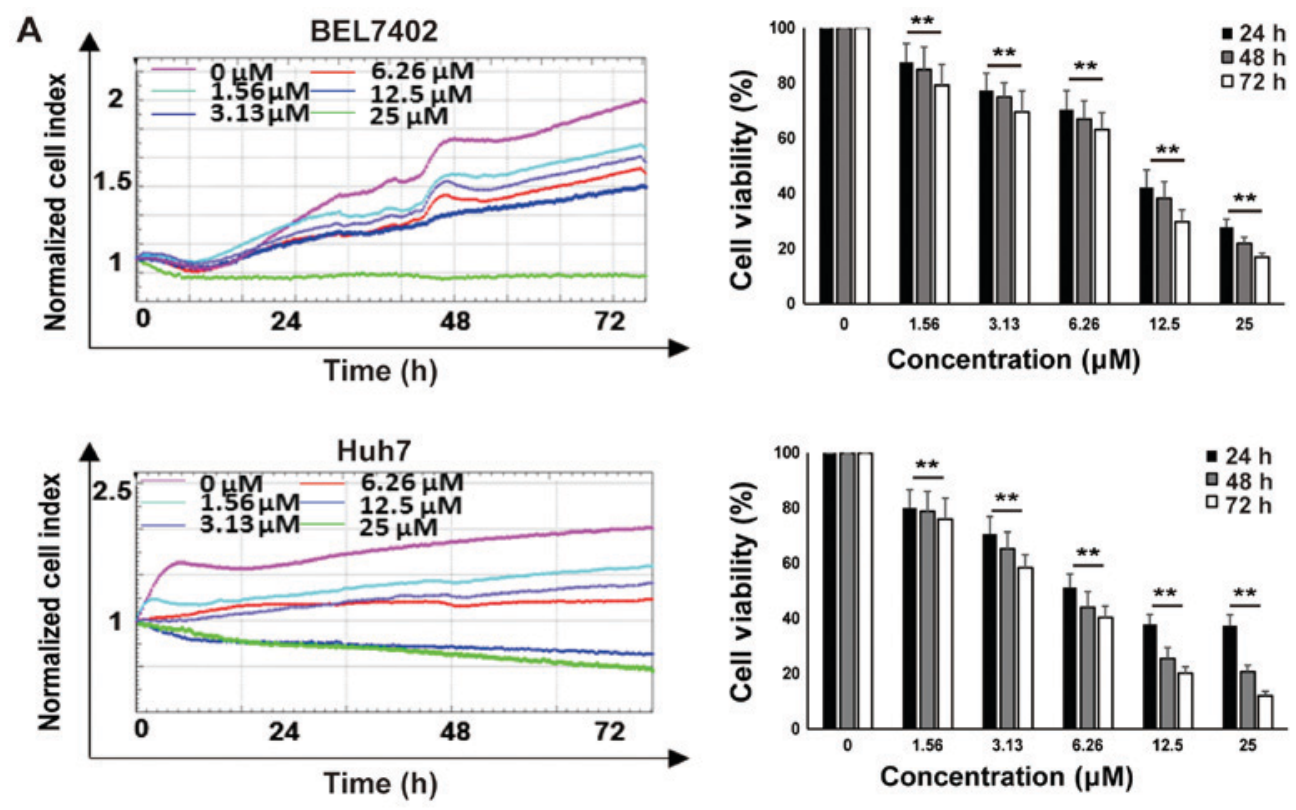
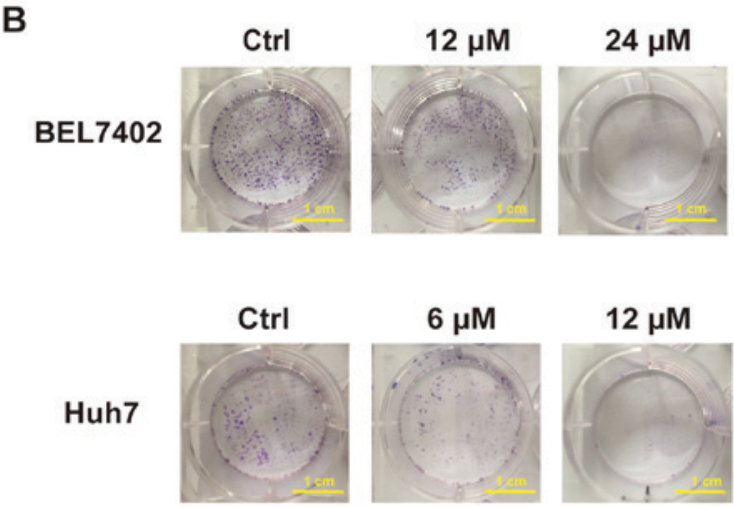

$12 \mu \mathrm{M}$

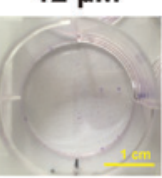

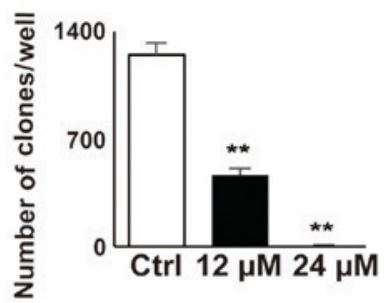

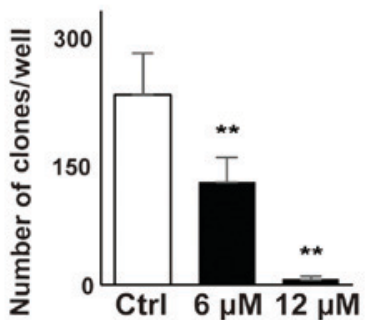

Figure 1. Vacq suppressed human HCC cell proliferation and colony formation. (A) 2x10 ${ }^{4}$ BEL7402 and Huh7 cells were seeded in an E-plate and treated with different concentrations of Vacq $(0,1.56,3.13,6.25,12.5$ and $25 \mu \mathrm{M})$ for $72 \mathrm{~h}$. Cell proliferation rates were monitored every 1 min and the results were quantified by xCELLigence RTCA system. All of the values were normalized to the cell indexes at the beginning of Vacq treatment and were presented as the fraction relative to cell indexes from the control group (set as 100\%). (B) BEL7402 cells were exposed to Vacq at 12 and $24 \mu \mathrm{M}$ respectively and Huh7 cells were at 6 and $12 \mu \mathrm{M}$ for $24 \mathrm{~h}$, and cultured in complete medium for 14 days. The number of the colonies was counted (scale bars, $1 \mathrm{~cm}$ ). Three independent experiments were performed. Data are presented as mean \pm standard error mean. ${ }^{* *} \mathrm{P}<0.01$ vs. control/as indicated. HCC, hepatocellular carcinoma; Vacq, Vacquinol-1; RTCA, Real-Time Cell Analyzer; Ctrl, control.

viability, BEL7402 and Huh7 cells were seeded in E-Plate 96, and cell proliferation was dynamically monitored by RTCA system for $72 \mathrm{~h}$. DMSO-treated HCC cells were used as controls. As shown in Fig. 1A, HCC cell proliferation was inhibited in a dose dependent manner. The $\mathrm{IC}_{50}$ of Vacq for BEL7402 at 24, 48 and $72 \mathrm{~h}$ was $12.35,11.48$ and $10.21 \mu \mathrm{M}$ and for Huh7 cells was 6.21, 5.58 and $4.89 \mu \mathrm{M}$ respectively. Next, to investigate the inhibitory effect of Vacq on HCC cellular growth potential, colony formation assay was performed. BEL7402 and Huh7 cells were exposed to Vacq at different concentrations for $24 \mathrm{~h}$ and then cultured in complete medium for 14 days. As depicted in Fig. 1B, the clonogenic abilities of HCC cells were significantly inhibited by Vacq. Together, these results indicated that Vacq suppresses human HCC cell viability in vitro.
Vacq induces human HCC cell apoptosis. To determine whether the inhibitory effect by Vacq on human HCC cells was due to apoptosis, BEL7402 cells were exposed to Vacq for $24 \mathrm{~h}$ and then analyzed using an Annexin V/PI double staining assay. DMSO-treated HCC cells were used as negative controls. Doxorubicin (Dox) was reported to induce HCC cell apoptosis (6). Therefore, $2 \mu \mathrm{M}$ Dox-treated cells were used as positive controls. As presented in Fig. 2A, the number of cells positive for either Annexin $\mathrm{V}^{+} \mathrm{PI}^{-}$(early apoptosis) or Annexin $\mathrm{V}^{+} \mathrm{PI}^{+}$(late apoptosis) was increased in Vacq-treated cells compared to DMSO-treated cells, suggesting that Vacq induces human HCC cell apoptosis. The apoptotic effect induced by Vacq in HCC cells was further assessed with Hoechst 33342 staining by fluorescence microscopy. As depicted in Fig. 2B, nuclear fragmentations and apoptotic 
A

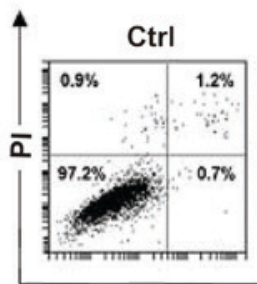

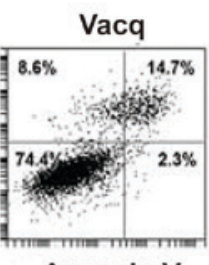

Annexin V

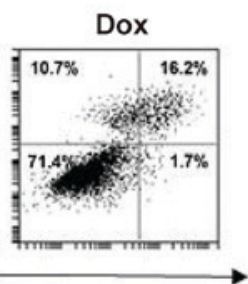

Vacq

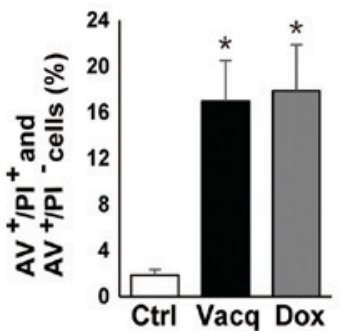

B
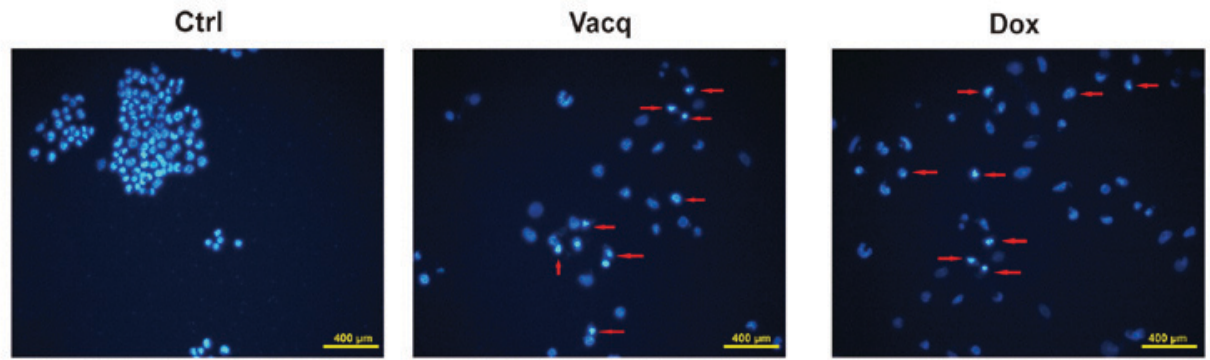

C
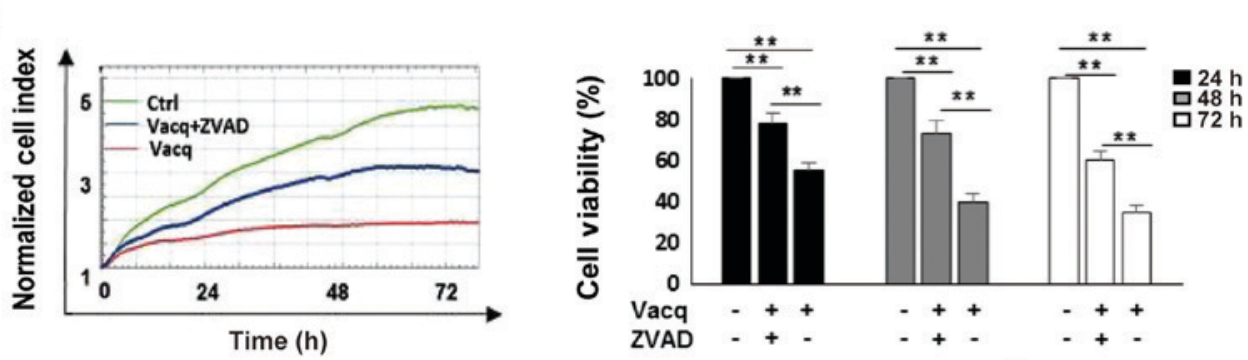

D
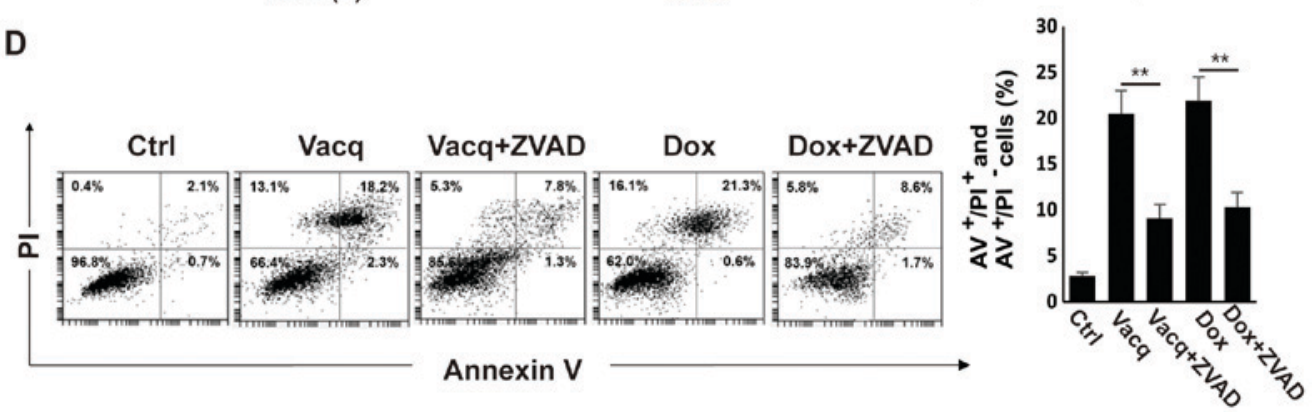

Figure 2. Vacq induces human HCC cell apoptosis. (A) BEL7402 cells were treated with Vacq at $10 \mu \mathrm{M}$. Following 24 h, HCC cells were double stained with Annexin V and PI. A total of $2 \mu \mathrm{M}$ Dox-treated cells were used as positive controls. Apoptotic cells corresponding to Annexin ${ }^{+} \mathrm{PI}^{-/+}$were quantified by flow cytometry. ${ }^{*} \mathrm{P}<0.05$ vs. Ctrl. (B) A total of $10 \mu \mathrm{M}$ Vacq was used to treat the BEL7402 cells for $24 \mathrm{~h}$. HCC cells were stained with Hoechst 33342 and observed by fluorescence microscope. The red arrows indicate nuclear morphologies (scale bars, $400 \mu \mathrm{m}$ ). BEL7402 cells were exposed to Vacq at $10 \mu \mathrm{M}$ in the presence or absence of $50 \mu \mathrm{M}$ ZVAD. (C) Cell viabilities were monitored by the xCELLigence RTCA system for $72 \mathrm{~h}$. (D) Following $24 \mathrm{~h}$ treatment, apoptosis was detected by flow cytometry. Three independent experiments were performed. Data are presented as the mean \pm standard error mean. ${ }^{* *} \mathrm{P}<0.01$, as indicated. Vacq, Vacquinol-1; Dox, Doxorubicin; AV, Annexin V; ZVAD, Z-VAD-FMK; Ctrl, Control; HCC, hepatocellular carcinoma; PI, propidium iodide.

bodies were observed in Vacq-treated HCC cells whereas the control cells displayed round and normal nuclei. To examine whether Vacq-initiated apoptosis is caspase-dependent, HCC cells were pretreated with a broad specificity caspase inhibitor, Z-VAD-FMK, and apoptosis was analyzed by flow cytometry. Pretreatment with Z-VAD-FMK substantially attenuated the reduction of cell viability compared to cells treated with Vacq alone (Fig. 2C). Fig. 2D shows that apoptosis was inhibited in Z-VAD-FMK pre-treatment group compared with the group exposure to Vacq alone. Altogether, these results suggested that Vacq induces apoptosis in HCC cells.

Vacq induces intrinsic apoptosis in HCC. Data from Fig. 2 suggested that Vacq might induce capase-depedent apoptosis in HCC cells. We then analyzed whether Vacq triggered caspase activation. Caspases, along with the poly(ADP-ribose) polymerase-1 (PARP-1) cleavage are characteristic features of apoptosis (7). Compared with the control groups, robust accumulations of cleaved caspase-3, caspase- 9 and PARP-1 but not caspase- 8 were observed in HCC cells exposure to Vacq. We further investigated the expression levels of the apoptosis related proteins in Vacq-treated HCC cells. Bcl-2 family which consist of both anti-apoptotic and pro-apoptotic proteins involves in the regulation of apoptotic cell death (8). Vacq exposure upregulated the pro-apoptotic proteins Bax and Bim while it downregulated the pro-survival protein $\mathrm{Bcl}-2$. The same protein expression patterns were observed in Dox-treatment groups which were used as positive control (Fig. 3A).

Bax protein was reported to translocate from the cell cytosol to the mitochondria during intrinsic apoptosis (9). To investigate whether Vacq induces Bax mitochondrial redistribution, Bel7402 cells were treated with Vacq for different 


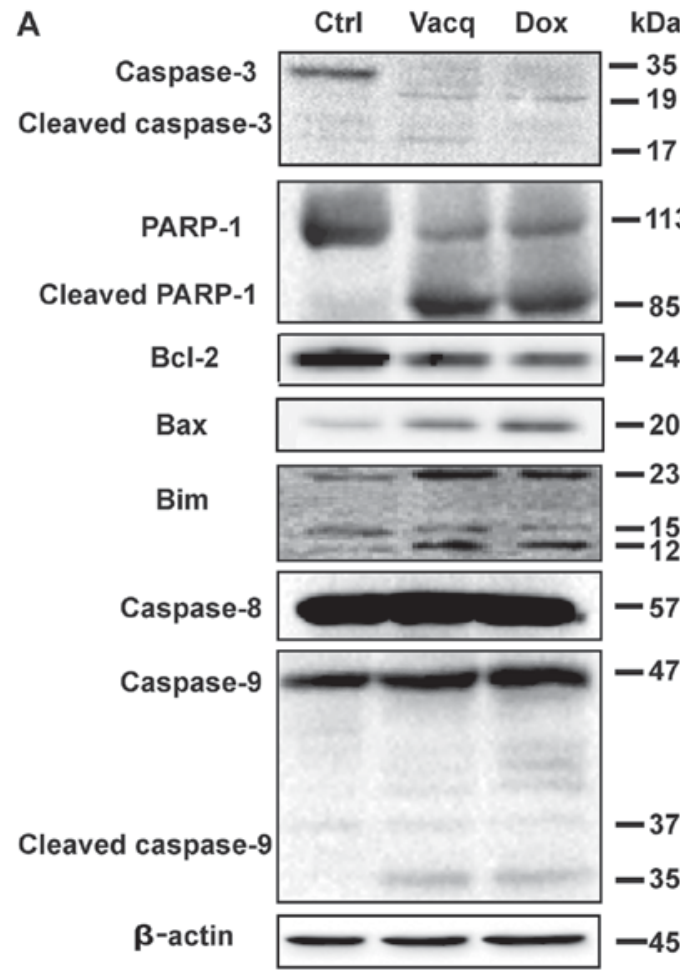

B

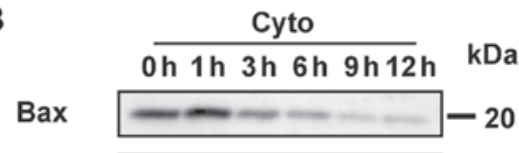

$\beta$-actin
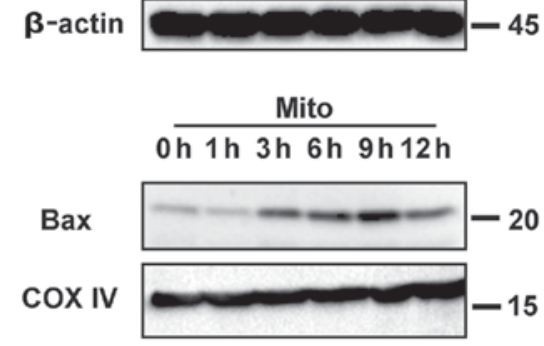

Figure 3. Vacq induces intrinsic apoptosis in HCC. (A) A total of $10 \mu \mathrm{M}$ Vacq was used to treat the BEL7402 cells for $24 \mathrm{~h}$. Caspase-3, caspase-8, caspase-9, PARP-1, BCL-2, Bax and Bim expression were detected by western blotting, using $\beta$-Actin as the loading control. (B) BEL7402 cells were treated with Vacq for the indicated time. The expression of cytosolic Bax and mitochondrial Bax were detected by western blotting. COX-IV and $\beta$-Actin were used as mitochondria and cytosol loading controls, respectively. Three independent experiments were performed. Vacq, Vacquinol-1; HCC, hepatocellular carcinoma; Dox, Doxorubicin; Ctrl, Control; PARP-1, poly(adenosine diphosphate-ribose) polymerase-1; BCL-2, B-cell lymphoma 2; Bax, Bcl-2-associated X protein; Bim, Bcl-2-like protein 11; Cyto, cytosol; Mito, mitochondria.

A

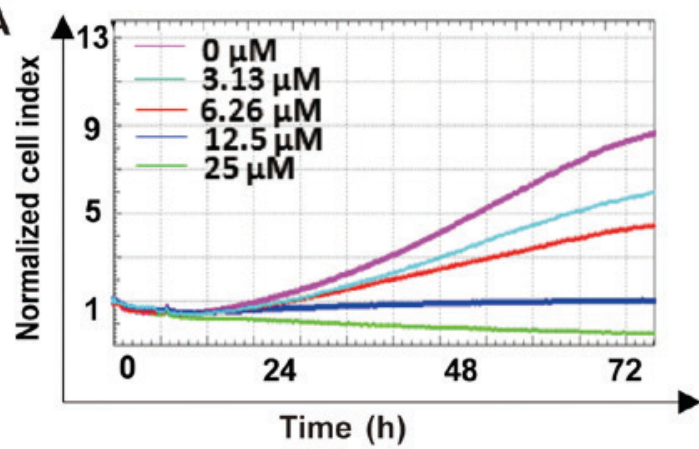

B

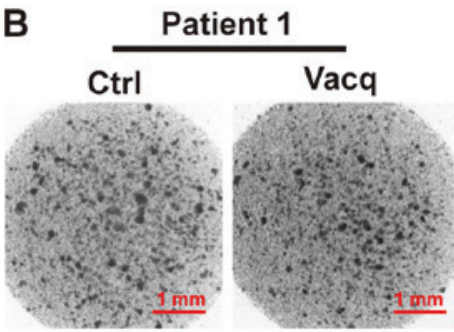

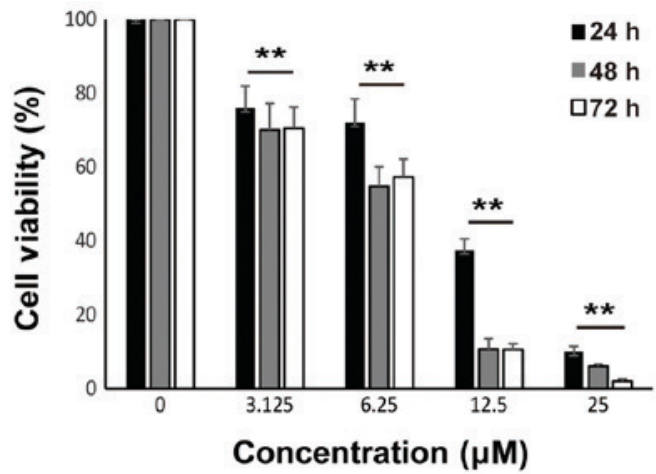

Concentration $(\mu \mathrm{M})$

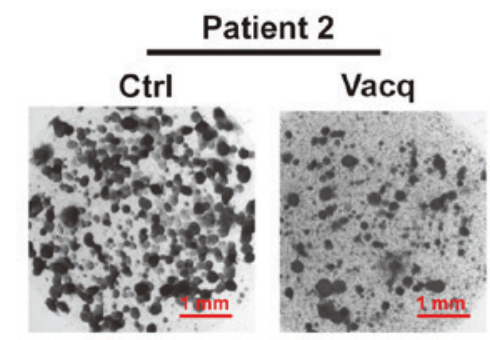

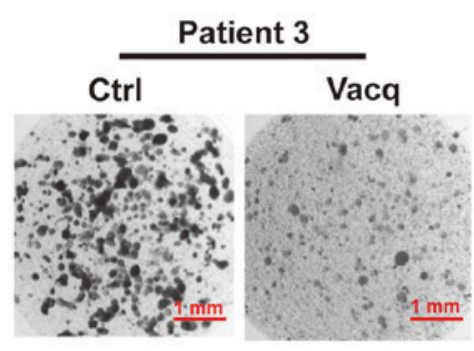

Figure 4. Vacq inhibited patient-derived HCC cell growth. (A) Patient-derived HCC cells were exposed to Vacq for $72 \mathrm{~h}$ at $3.13,6.25,12.5$ and $25 \mu \mathrm{M}$, respectively. Cell viability was monitored by xCELLigence RTCA system every $1 \mathrm{~min}$. Data are presented as mean \pm standard error mean from 10 different individuals. ${ }^{* *} \mathrm{P}<0.01$, as indicated. (B) The sensitivity to Vacq was further investigated by using collagen gel droplet embedded culture-drug sensitivity test. Patient-derived HCC cells were cultured in collagen gel droplets and exposed to $15 \mu \mathrm{M}$ Vacq. Cell viabilities were quantified by staining with neutral red and detected by image analysis. The results shown are representative from 3 individuals (scale bars, $1 \mathrm{~mm}$ ). Vacq, Vacquinol-1; HCC, hepatocellular carcinoma; Ctrl, Control.

durations, and the expressions of Bax proteins in cytosol and mitochondria fractions were assessed by western blot, respectively. As depicted in Fig. 3B, Vacq induced the increase of mitochondria Bax expression, combined with the significant 
depletion of Bax in the cytosol compartment. Taken together, these results indicated that Vacq induced intrinsic apoptosis in HCC.

Vacq inhibited patient-derived HCC cells proliferation. To evaluate the clinical translational significance of Vacq, we examined the antitumor effects of Vacq in patient-derived HCC cells. Cells from 10 different individuals were exposed to Vacq for $72 \mathrm{~h}$ at $3.13,6.25,12.5$ and $25 \mu \mathrm{M}$, respectively. DMSO was used as control. As shown in Fig. 4A, cell growth was inhibited by Vacq in a dose-dependent manner. The average IC50 of these 10 individuals at 24, 48 and $72 \mathrm{~h}$ were $10.7,8.9,7.4 \mu \mathrm{M}$, respectively. The sensitivities of these cells to Vacq were further assessed by collagen gel droplet embedded CD-DST, an in vitro anticancer drug sensitivity test in which the reported clinical correlation was approximately $91 \%$ in several solid cancers $(10,11)$. Patient-derived HCC cells were packaged into the collagen droplets to provide a 3-D culture environment and treated with Vacq at $15 \mu \mathrm{M}$ for $24 \mathrm{~h}$. Viable cells were deeply stained by neural red which presented in dark color in Fig. 4B. Three results shown in Fig. 4B. were represented to the patients who were insensitive (patient 1) or sensitive (patient 2 and 3) to Vacq. In sensitive group, compared with DMSO treatment, Vacq-treated cells were substantially stained, which related to the inhibitory effect of Vacq in patient tumor-derived HCC cells.

\section{Discussion}

Vacq has been reported to induce the death of human malignant glioma cells without affecting normal cells $(3,12)$. We here demonstrate that Vacq triggers apoptosis in both HCC cell lines as well as in HCC patients-derived primary hepatoma cells. To the best of our knowledge, this study has been the first to identify that Vacq inhibits HCC cell growth. Thus, our data may shed new lights into chemotherapy of HCC patients.

Previous study showed that Vacq induces GCs massive macropinocytic vacuolization which was a novel form of cell death termed as methuosis (13). Massive macropinocytic vacuole accumulation leads to the rupture of GC cytoplasmic membrane and cell death. Despite of caspase-3/7 activation, the GC death triggered by Vacq treatment was morphologically distinct from apoptosis. Moreover, pretreatment with caspase inhibitor zVAD-FMK only delayed the Vacq-induced GC cell death and $100 \%$ cell death occurred later (12). These data suggest that Vacq induced non-apoptotic cell death in GC. In addition, sensitivity to macropinocytosis is a unique cellular property of GCs $(14,15)$. Therefore, the antitumor mechanism of vacq on other cancers needs to be further explored.

In the present study, we show that Vacq induces human HCC cell death which may be mediated through apoptosis. Flow cytometry analysis revealed that Vacq increased both Annexin $\mathrm{V}^{+}$ $\mathrm{PI}^{-}$and Annexin $\mathrm{V}^{+} \mathrm{PI}^{+}$population cells whereas the caspase inhibitor Z-VAD-FMK efficiently decreased the apoptosis cells, suggesting induction of apoptosis in Vacq-treated HCC cells. This notion was further supported by the morphological observation of the nuclear fragment formation in HCC cells exposure to Vacq. In addition, Vacq treatment activated both caspase-3, caspase-9 and PARP-1 cleavage, upregulated the expressions of Bim and downregulated pro-survival protein
Bcl-2 expression in HCC cells. Furthermore, Vacq treatment induced Bax translocation to mitochondria. Taken together, Vacq induces HCC cell death in an intrinsic apoptosis-dependent manner, which is distinct from the massive macropinocytic vacuolization in Vacq-treated glioma cells. Further studies necessary to elucidate the exact underlying mechanism of the antitumor effects of Vacq on HCC.

Notably, we also demonstrate that Vacq suppressed patient-derived HCC cell growth in both two-dimensional (2D) and three-dimensional (3D) cultures, suggesting of clinical translational significance for the treatment of HCC. Further study should include toxicity studies on a broad range of normal human liver cells to confirm the selectivity of Vacq towards HCC cells. In conclusion, the results of the present study provide preliminary evidence that Vacq displays potent inhibitory effects on HCC cell growth. Our data warrant further investigation of Vacq as a novel antitumor compound for the treatment of HCC.

\section{Acknowledgements}

The authors would like to thank Dr Songshu Meng (Institute of Cancer Stem Cell, Dalian Medical University Cancer Center, Dalian, China) for the technical advice.

\section{Funding}

The present study was supported by Liaoning Provincial Major Projects for Clinical Capacity-Building (grant no. LNCCC-B04-2015) and Liaoning Province Ph.D. Research Start-up Fund Project (grant no. 201501109).

\section{Availability of data and materials}

The datasets used and/or analyzed during the current study are available from the corresponding author on reasonable request.

\section{Authors' contributions}

SLS and HP conceived and designed the study. SLS was a major contributor in writing the manuscript. XL analyzed the data. NS and SC performed the experiments, including cell viability, colony formation and nuclear morphological observation. XG performed western blot analysis. GZ conducted the primary HCC cell sensitivity test. All authors read and approved the final manuscript.

\section{Ethics approval and consent to participate}

The present study was approved by the Medical Ethical Committee of the Liaoning Cancer Hospital (China; Ethics Review Approval no. 20160213-3). Written informed consent was provided by all of the participants.

\section{Consent for publication}

All patients, or their parent, guardian or next of kin, provided written informed consent for the publication of any associated data and accompanying images. 


\section{Competing interests}

The authors declare that they have no competing interests.

\section{References}

1. Firtina Karagonlar Z, Koc D, Iscan E, Erdal E and Atabey N: Elevated hepatocyte growth factor expression as an autocrine c-Met activation mechanism in acquired resistance to sorafenib in hepatocellular carcinoma cells. Cancer Sci 107: 407-416, 2016.

2. Villanueva A, Hernandez-Gea V and Llovet JM: Medical therapies for hepatocellular carcinoma: A critical view of the evidence. Nat Rev Gastroenterol Hepatol 10: 34-42, 2013.

3. Sander P, Mostafa H, Soboh A, Schneider JM, Pala A, Baron AK, Moepps B, Wirtz CR, Georgieff M and Schneider M: Vacquinol-1 inducible cell death in glioblastoma multiforme is counter regulated by TRPM7 activity induced by exogenous ATP. Oncotarget 8: 35124-35137, 2017.

4. Wang X, Sun D, Tai J and Wang L: Ganoderic acid A inhibits proliferation and invasion, and promotes apoptosis in human hepatocellular carcinoma cells. Mol Med Rep 16: 3894-3900, 2017.

5. Naitoh H, Yamamoto H, Murata S, Kobayashi H, Inoue K and Tani T: Stratified phase II trial to establish the usefulness of the collagen gel droplet embedded culture-drug sensitivity test (CD-DST) for advanced gastric cancer. Gastric Cancer 17 : 630-637, 2014.

6. Wang J, Ma L, Tang X, Zhang X, Qiao Y, Shi Y, Xu Y, Wang Z, Yu Y and Sun F: Doxorubicin induces apoptosis by targeting Madcam 1 and AKT and inhibiting protein translation initiation in hepatocellular carcinoma cells. Oncotarget 6: 24075-24091, 2015.

7. Los M, Mozoluk M, Ferrari D, Stepczynska A, Stroh C, Renz A, Herceg Z, Wang ZQ and Schulze-Osthoff K: Activation and caspase-mediated inhibition of PARP: A molecular switch between fibroblast necrosis and apoptosis in death receptor signaling. Mol Biol Cell 13: 978-988, 2002.
8. Czabotar PE, Lessene G, Strasser A and Adams JM: Control of apoptosis by the BCL-2 protein family: Implications for physiology and therapy. Nat Rev Mol Cell Biol 15: 49-63, 2014.

9. Gillies LA and Kuwana T: Apoptosis regulation at the mitochondrial outer membrane. J Cell Biochem 115: 632-640, 2014.

10. Kobayashi H, Tanisaka K, Doi O, Kodama K, Higashiyama M, Nakagawa H, Miyake M, Taki T, Hara S, Yasutomi M, et al: An in vitro chemosensitivity test for solid human tumors using collagen gel droplet embedded cultures. Int J Oncol 11: 449-455, 1997.

11. Miyazaki R, Anayama T, Hirohashi K, Okada H, Kume M and Orihashi K: In vitro drug sensitivity tests to predict molecular target drug responses in surgically resected lung cancer. PLoS One 11: e0152665, 2016.

12. Hammarström LG, Harmel RK, Granath M, Ringom R, Gravenfors $Y$, Färnegårdh $K$, Svensson $P H$, Wennman D, Lundin G, Roddis Y, et al: The oncolytic efficacy and in vivo Pharmacokinetics of [2-(4-Chlorophenyl)quinolin-4-yl] (piperidine-2-yl)methanol (Vacquinol-1) are governed by distinct stereochemical features. J Med Chem 59: 8577-8592, 2016.

13. Overmeyer JH, Young AM, Bhanot $\mathrm{H}$ and Maltese WA: A chalcone-related small molecule that induces methuosis, a novel form of non-apoptotic cell death, in glioblastoma cells. Mol Cancer 10: 69, 2011.

14. Shi Y, Lim SK and Parada LF: The soft underbelly of tumor cells. Cell Res 24: 910-911, 2014

15. Lim JP and Gleeson PA: Macropinocytosis: An endocytic pathway for internalising large gulps. Immunol Cell Biol 89 836-843, 2011. 